\title{
The Development of FMTVDM-BEST IMAGING@(D: The Answer for Breast Cancer. Breast Enhanced Scintigraphy Test (BEST@®): Quantifying the Detection of Breast Cancer and its Treatment.
}

\author{
Richard M Fleming ${ }^{1 *}$, William C Dooley ${ }^{2}$ and Tapan K Chaudhuri ${ }^{3}$ \\ ${ }^{1}$ The Camelot Foundation, Studio City, California, USA \\ ${ }^{2}$ Division of Surgical Oncology, Oklahoma University Health Science Center, Oklahoma, USA
}

${ }^{3}$ Department of Radiology, Eastern Virginia Medical School, USA

\begin{abstract}
Background: The diagnostic testing for breast cancer has been limited by the absence of a method, which can quantitatively differentiate tissue differences; specifically differentiation between calcium deposits, normal breast tissue, inflammatory changes in breast tissue and breast cancer. Such a quantitative method would remove the issue of sensitivity and specificity errors and allow for diagnostic decision-making including treatment monitoring.
\end{abstract}

Method: A series of investigations were conducted over an 18-year period of time looking at more than 1000 women and men suspected of having breast tissue abnormalities. The studies compared pathologic tissue results with outcomes using other diagnostic studies and FMTVDM-BESTO@ Imaging. The studies began with asking the fundamental questions necessary to produce such a quantitative diagnostic test, followed by correcting for errors encountered by other methods. Following the initial work comparing FMTVDM-BEST@® Imaging with other tests and tissue pathology results, the investigations turned to monitoring changes in women over time, demonstrating the ability to monitor treatment results from surgery, chemotherapy, radiation therapy, immune therapy, diet and lifestyle changes. Further work was conducted to assure that the time within a woman's cycle did not affect FMTVDM-BESTCP Imaging.

The next series of studies looked at the effect that soy protein, smoking and hormone treatment (HRT) had on breast tissue health and the relationship between breast cancer and these influences. The summation of all of these studies included looking at breast density and breast implants as well as male patients.

Results: FMTVDM-BESTOP Imaging quantitatively differentiates between breast calcium deposits, normal breast tissue, inflammatory changes and breast cancer. It can monitor transitions in tissue showing both the progression and regression of disease allowing clinicians to monitor treatment outcomes independent of the treatment approach used. FMTVDM-BESTOP Imaging is not affected by breast density, breast implants, mastectomy or size of breast; including male patients.

Conclusion: FMTVDM-BESTCP Imaging provides the first and only quantitative method, which can differentiate tissues based upon changes in measurements obtained from this patented method. As such, it can detect changes in tissue leading to breast cancer allowing for greater treatment opportunities, as well as monitoring outcomes of treatment for breast cancer and other abnormalities (e.g. fibrocystic disease). FMTVDM-BEST@® Imaging works independent of whether the patient has dense breast tissue, breast implants, is on HRT, smokes or has undergone previous treatment in both men and women.

Keywords: Diagnostic testing; Breast cancer; Imaging; Breast Enhanced Scintigraphy Test

\section{Introduction}

In 1999, a representative from the pharmaceutical company owning Sestamibi approached me to determine if I would be willing to study breast cancer using Sestamibi. At that time, I was working on "Inflammation and Heart Disease" (c) and the diagnostic evaluation of ischemia. So in 1999 we began the development of a quantitative method, which could accurately use nuclear imaging to quantify both (1) heart disease and (2) breast cancer. The stipulated requirement provided that we would only study people with a clinical question of having both heart disease and breast cancer.

\section{Materials and Methods}

The work began by asking if we could develop a test, which could specifically identify breast cancer. After identifying the two critical properties of blood flow and metabolism, the factors, which critically distinguish cancer from inflammation from normal breast tissue from calcium, the second task was to determine if there was a method, which could "enhance" those differences briefly. Using methods commonly employed in Cardiology, we were able to develop such a diagnostic test, which could pharmacologically enhance these differences. The third and final question remained as to whether we could accurately measure such differences and after identifying problems with modulation transfer function (MTF) and Fourier Transform (FT), we were able to correct for the loss of $50 \%$ of the data, which we were able to determine using known samples of technetium 99-m and measuring decay curves.

*Corresponding author: Richard M Fleming, The Camelot Foundation, Studio City, CA, USA, Tel: 818-209-1820; E-mail: rmfmd7@yahoo.com

Received December 05, 2017; Accepted December 12, 2017; Published December 18, 2017

Citation: Fleming RM, Dooley WC, Chaudhuri TK (2017) The Development of FMTVDM-BEST IMAGING@P: The Answer for Breast Cancer. Breast Enhanced Scintigraphy Test (BEST(O)): Quantifying the Detection of Breast Cancer and its Treatment. J Nucl Med Radiat Ther 8: 350. doi: 10.4172/2155-9619.1000350

Copyright: @ 2017 Fleming RM, et al. This is an open-access article distributed under the terms of the Creative Commons Attribution License, which permits unrestricted use, distribution, and reproduction in any medium, provided the original author and source are credited. 
Citation: Fleming RM, Dooley WC, Chaudhuri TK (2017) The Development of FMTVDM-BEST IMAGING@®: The Answer for Breast Cancer. Breast Enhanced Scintigraphy Test (BESTOP): Quantifying the Detection of Breast Cancer and its Treatment. J Nucl Med Radiat Ther 8: 350. doi: 10.4172/2155-9619.1000350

Page 2 of 8

The development of FMTVDM-BEST IMAGING@® (BEST@®) began with a simple inclusion of breast imaging using a SPECT camera in planar mode having an intrinsic resolution of $3.4 \mathrm{~mm}$, a $128 \times 128$ matrix and a LEHR collimator. Under these conditions, we imaged our first lady in the spring of 1999. Her result is shown in Figure 1. We initially displayed the result qualitatively, using the customary black and white image. The initial response by the pharmaceutical representative was one of apprehension and concern that the image was a false positive. Our first change, seen to the right, was to change the image to a bluegreen image, which allows for better human visual interpretation. To her delight, the false positive irregularities disappeared.

The observation of this qualitative error, like that which we had noticed in cardiac imaging (FMTVDM-FHRWW (OP), resulted in the decision that these studies needed to be "quantified." Since the images are formatted using the radioactive counts obtained by the photomultiplier tubes, the software required to do this "quantification" already existed deep within the programs from decades before when regions of interest (ROIs) were looked at. As a nuclear cardiologist, I had decided that for me to investigate the use of Sestamibi and later other isotopes for breast cancer would require that I initially only look at women and men, who had both an issue of ischemia and cancer. Since the uptake and subsequent "quantification" of Sestamibi is dependent upon both blood flow and mitochondrial function, the first question, was to compare differences between standard breast cancer studies (Miraluma) and the enhanced delivery of Sestamibi using either exercise or pharmacologic means, to augment delivery of the isotope by enhancing the blood flow found in cancers compared to "normal" or "inflammatory" breast tissue. The two significant differences between these types of tissue was both the difference in blood supply (normal<inflammatory tissue with increased blood flow $<$ cancers) going to these tissues and the concentration of mitochondria (mitochondrial activity) within [dead (e.g. myocardial infarction or calcium deposits) $<$ normal $<$ inflammatory leukocytes $<$ cancer] more highly metabolic tissues.

Standard Miraluma (the resting, non-enhanced approach) is performed using the same camera settings laid out above and the same timing used for our enhanced BEST@ $\odot$ method as shown in Figure 2 , minus the enhancement component. As stated BEST@ $\odot$ enhances the delivery of isotope to breast tissue by augmenting differential blood

\section{Side by Side Comparison}

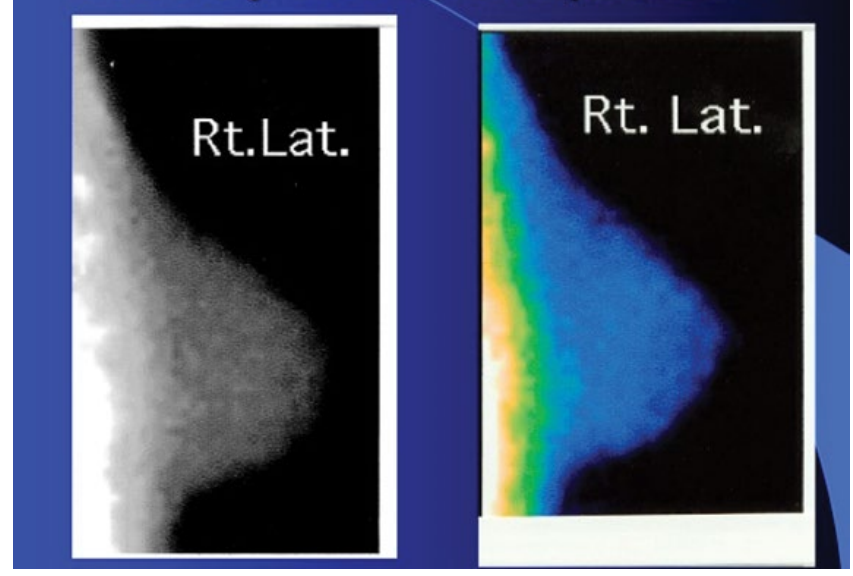

Figure 1: Elimination of qualitative visual errors by converting image from blackwhite to blue green.

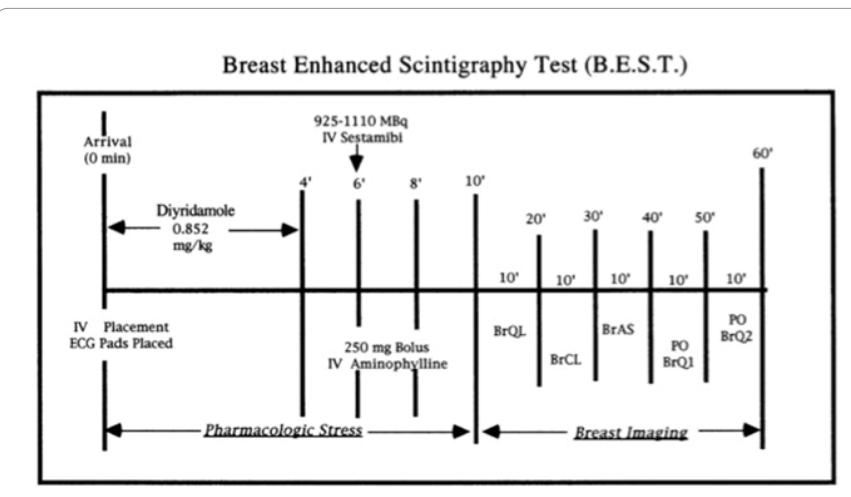

Figure 2: Enhanced delivery of the isotope was obtained by vasodilation of blood vessels to breast tissue using Dipyridamole (or applicable agent or exercise), with the introduction of Sestamibi through an intravenous catheter at peak vasodilator effect (6 minutes). Aminophylline was used if indicated and imaging began with the breast in question (BrQL) and then the contralateral $(\mathrm{BrCL})$ breast. This initial method also imaged both breasts anteriorly (BrAS) and provided for additional imaging if further questions (BrQ1, BrQ2) existed after the earlier image acquisition.

flow present in breast tissue, depending upon the vascularity present in different tissue types, as described in Figure 2 [1]. Quantification of isotope delivery and uptake was then drawn using ROIs as shown in Figure 3.

\section{Results and Discussion}

Both Miraluma and B.E.S.T. were compared with multiple biopsy specimens obtained from 10 women studied using both methods. The results shown in Figure 4, showed no statistically significant difference in the results obtained in "normal" breast tissue using either method as there is no vascular or metabolic differences to enhance, while B.E.S.T. had a statistically significantly $(\mathrm{p}<0.05)$ higher "quantified" uptake of Sestamibi than did Miraluma within inflammatory changes in breast tissue and an even greater difference $(\mathrm{p}<0.005)$ in "quantifiable" uptake of Sestamibi in breast cancer compared with Miraluma.

Between February of 1999 and June of 2005, a total of 992 women and 4 men were studied using BEST@® imaging. The studies included determinations of differences between normal breast tissue, inflammatory breast tissue (fibrocystic disease), atypia (early tissue change) and breast cancer [2-8]. The method of "quantification" allowed for the average (mean) \pm standard deviation present, as well as the minimum and maximum count activity within the entire breast and within localized regions of the breast as shown in figure 3. In the end, the greatest value was the "Maximal Count Activity (MCA)" which represents the most metabolically active region with the greatest enhancement of blood flow to the breast, as shown in Figure 4 . The variance of MCA and changes over time demonstrates whether there is a progression or regression of disease. Further camera settings, software and specific protocol and analysis of the findings are a matter of patent licensure as is this method.

Given the differentiation of the statistically significant clinical utility of BEST@(P) imaging, we turned our attention to comparing the results of BEST $\odot$ (P) and tissue specimens with that of mammography. In comparing 100 women, we were delighted to have the additional diagnostic support of several surgeons including Dr. William Dooley, who provided biopsy and ductoscopy information in some of the women. The additional value of this work emphasizes the value of his ductoscopy procedure for obtaining tissue without breast incision, 
Citation: Fleming RM, Dooley WC, Chaudhuri TK (2017) The Development of FMTVDM-BEST IMAGING@®: The Answer for Breast Cancer. Breast Enhanced Scintigraphy Test (BESTOP): Quantifying the Detection of Breast Cancer and its Treatment. J Nucl Med Radiat Ther 8: 350. doi: 10.4172/2155-9619.1000350

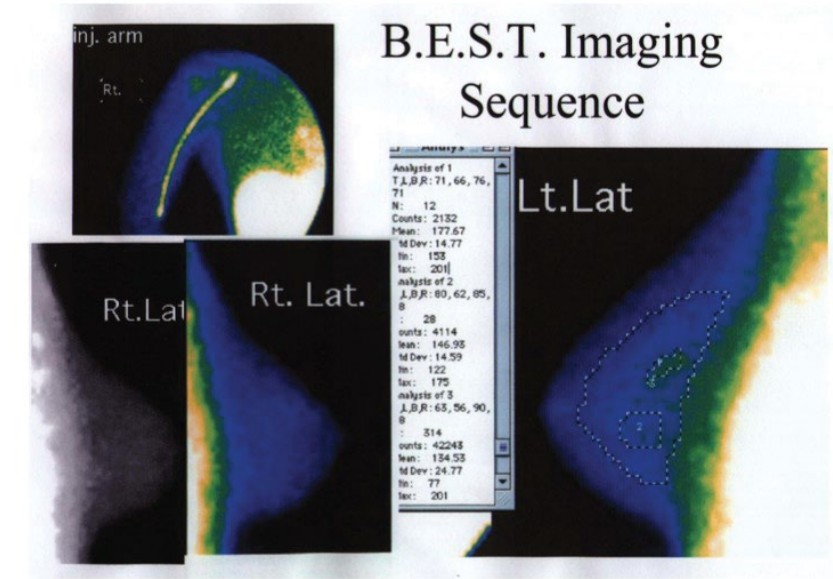

Figure 3: ROIs are drawn for each breast image and "quantified" results were recorded. ROls were drawn in the same manner for Miraluma images recording the "quantified" results.

\section{A Comparison of Miraluma and Breast Enhanced Scintigraphy.}

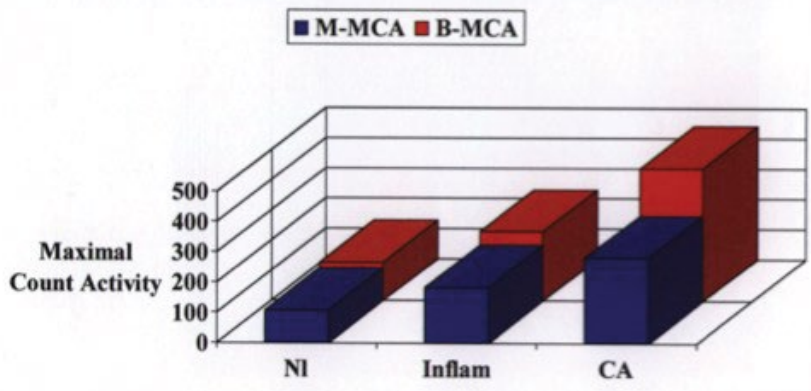

Figure 4: "Quantified" differences in Sestamibi uptake using B.E.S.T. and Miraluma where undetectable in "normal" tissue, but statistically different $(p<0.05)$ in inflammatory and cancerous $(p<0.005)$ tissue as noted in the bar graph.
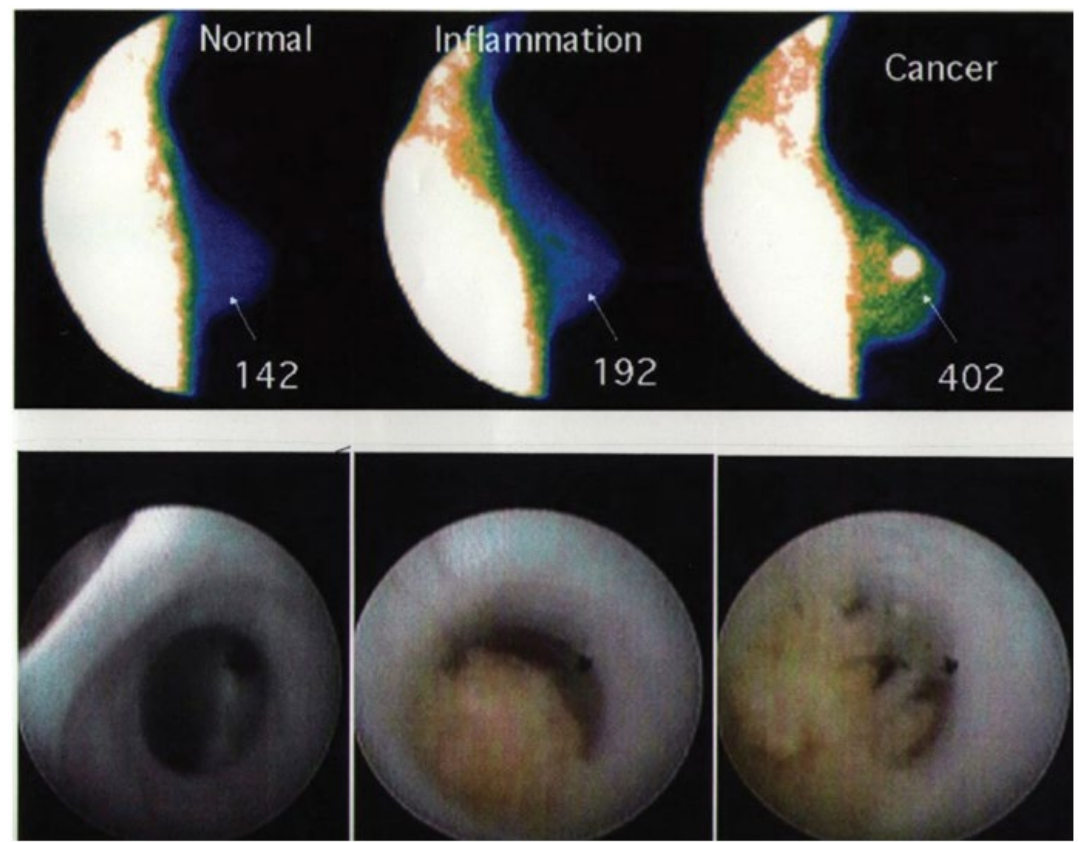

Figure 5: Three different women who had tissue specimens obtained via ductoscopy and their respective tissue results and MCA values obtained via B.E.S.T. imaging.

to compare with BEST@ $\odot$ and mammography. An example of a comparison of ductoscopy and BEST@P is shown in Figure 5.

When the results of MCA were analyzed and graphically displayed, the normal breast tissue showed MCA values of $144 \pm 30$ with a $95 \%$ confidence (CI) interval from 138 to 150 . Inflammatory tissue with its greater mitochondrial activity and blood supply revealed MCA values of $229 \pm 50$ with a $95 \%$ CI from 219 to 240 , while the still higher metabolic (mitochondria) activity with associated angiogenesis typifying cancers had MCAs of $446 \pm 80$ and a $95 \%$ CI from 404 to 489 . These results are shown in Figure 6 with bar graph comparisons of the three shown in Figure 7.
Given information showing separation in MCA between normal, inflammatory and cancerous breast tissue, with evidence to indicate that this progression of tissue change is associated with an overlap at the extremes of MCA where tissue differentiation occurs, we next turned our attention to looking for evidence of atypia, which would further suggest a transition from inflammatory tissue (fibrocystic disease, FCD) to breast cancer, which could be measured by BEST@(P MCA values. To do this, we studied 201 women and 4 men and as previously discussed, matched the normal, FCD and CA tissue with MCA counts and generated the results seen in Figure 8 [3].

An example of a specific patient showing these changes in MCA 
Citation: Fleming RM, Dooley WC, Chaudhuri TK (2017) The Development of FMTVDM-BEST IMAGING@®: The Answer for Breast Cancer. Breast Enhanced Scintigraphy Test (BESTOP): Quantifying the Detection of Breast Cancer and its Treatment. J Nucl Med Radiat Ther 8: 350. doi: 10.4172/2155-9619.1000350

Page 4 of 8

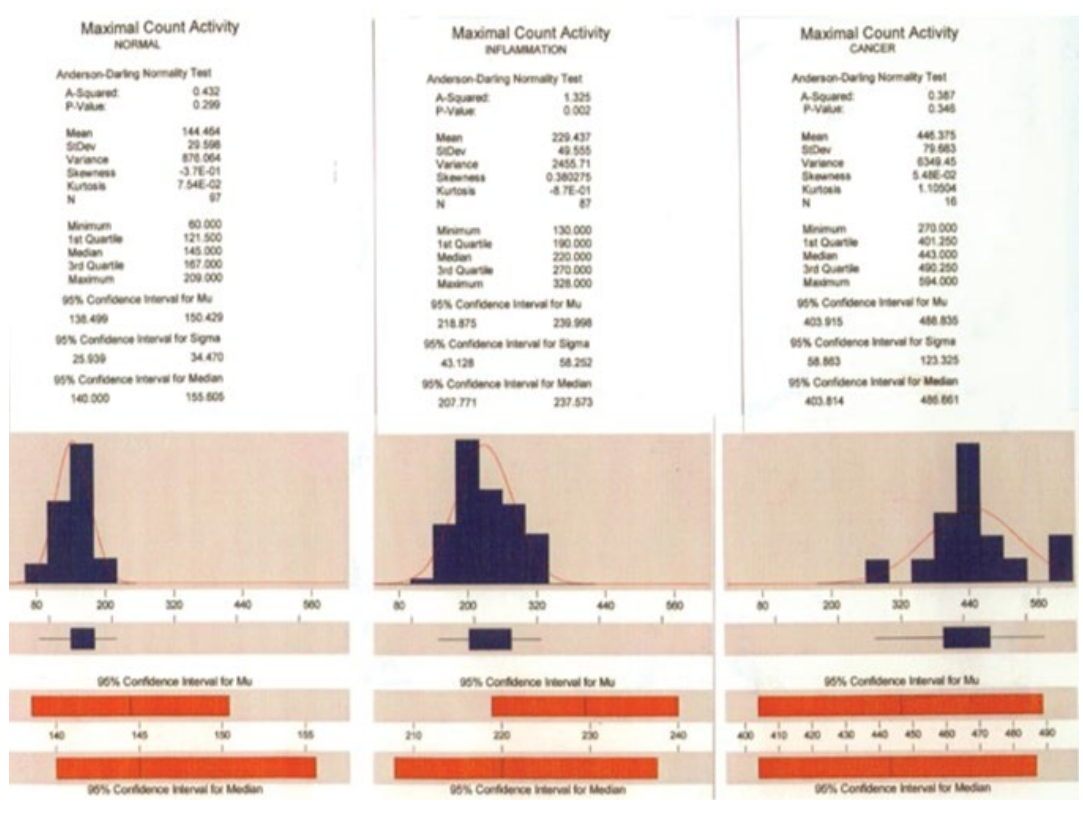

Figure 6: The distribution of MCA values for normal, inflammatory breast tissues and cancer.

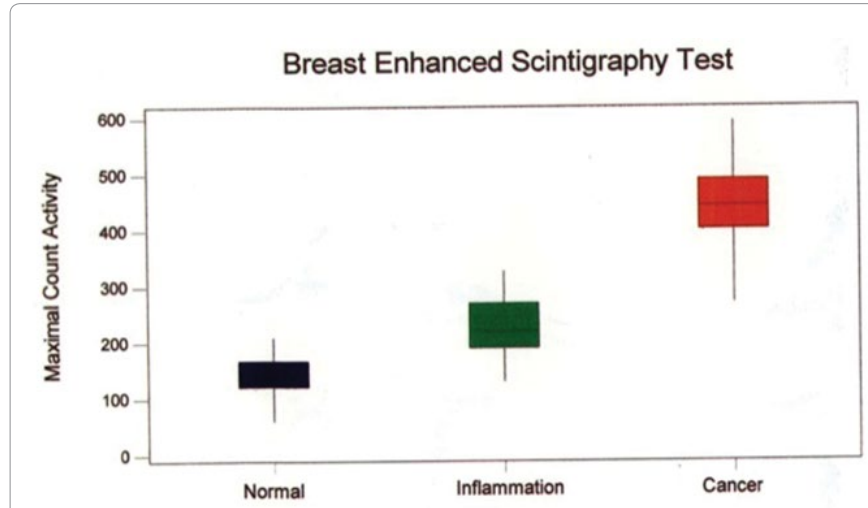

Figure 7: Graphic depiction of MCA values with $95 \% \mathrm{Cl}$ noted by the horizonta lines within the colored boxes with the boxes representing the $95 \% \mathrm{Cl}$. The vertical lines represent all values seen demonstrating the transition as breast tissue changes from "normal" to "inflammatory" to "cancer."

over time is shown in Figure 9. As noted, these changes confirmed by biopsy were not associated with a cancerous mass, revealing the importance of detection of proliferation changes in breast tissue, long before the development of a mass which only then might have been detectable by anatomic mass tests like mammography, CT or MRI. Further investigation of the results allowed further comparisons including cellular atypia are shown in Figure 10. Changes in tissue proliferative changes are demonstrated by increasing MCA values on B.E.S.T○(P Imaging as tissue changes from normal $(145.0 \pm 29.1)$ to inflammatory $(218 \pm 40.3)$ to atypia/metaplasia $(307.7 \pm 29.3)$ to cancer $(445.3 \pm 83.3)$. An example of one of the male participants with breast cancer is shown in Figure 11.

Additional information obtained in this study unmasked the metabolic effects of larger cancers on smaller cancers. In Figure 12, a woman studied twice in 9 months, showed a growth in the size of her primary breast cancer in her right breast with an MCA going from 425

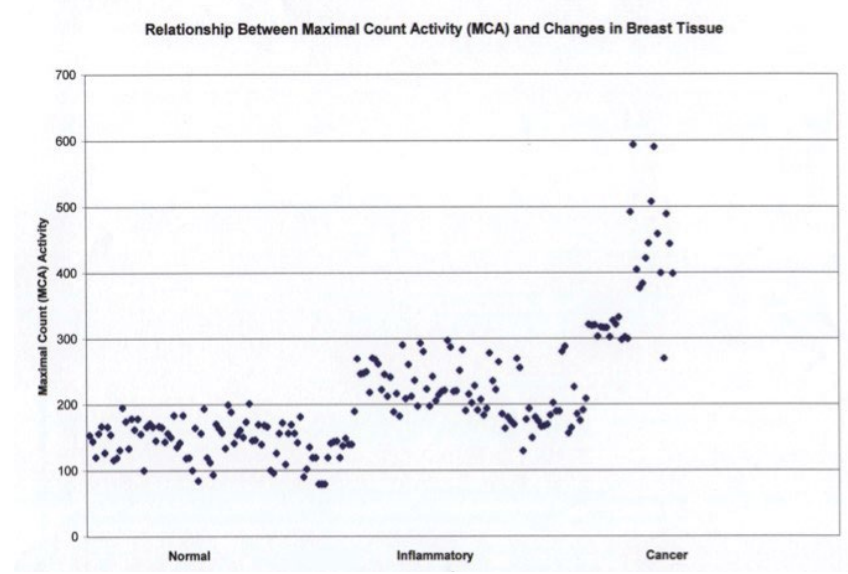

Figure 8: Changes in MCA demonstrate that there is an exponential change in tissue blood flow and metabolic (mitochondria) activity as breast tissue changes from "normal" to FCD to cancer, with a region between FCD and CA suggesting further differentiation.

to 470 , while the MCA of the left breast went from 240 to 185 . Within 6 months after having the right breast cancer removed, a new cancer was detected in the left breast, which was surgically removed. These findings indicate that larger cancers can inhibit the growth of smaller cancers through "inhibitors" as posted by the other components of FMTVDM๑®.

Given the ability to differentiate the proliferative changes that occur as normal breast tissue transitions to breast cancer and our ability to detect the inhibitory effect that larger primary cancers can have on smaller slower growing secondary cancers, we began to ask if we could measure treatment effects. We began by looking at the effects of soy protein on inflammatory breast disease [4,5]. These 64 women had fibrocystic disease, a common problem among women which can 
Citation: Fleming RM, Dooley WC, Chaudhuri TK (2017) The Development of FMTVDM-BEST IMAGING@®: The Answer for Breast Cancer. Breast Enhanced Scintigraphy Test (BESTOD): Quantifying the Detection of Breast Cancer and its Treatment. J Nucl Med Radiat Ther 8: 350. doi: 10.4172/2155-9619.1000350

\section{Ductal Carcinoma In-Situ}

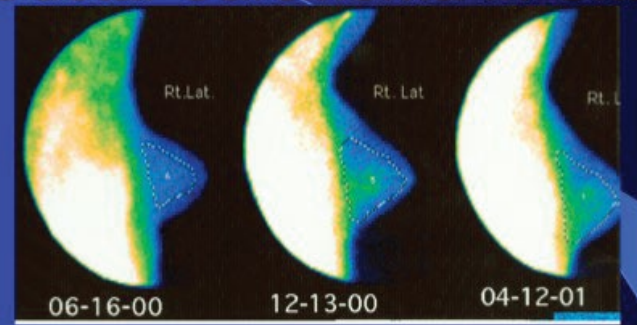

The initial MCA on 6-16-2000 was 142.

On 12-13-2000 the MCA was 165.

In April of 2001, the MCA was 240.

The Biopsy performed 20 June 2001 showed DCIS.

Figure 9: An example of how rapidly normal breast tissue can progress from "normal" to DCIS is shown in this patient, whose MCA values increased from 142 to 165 in 6 months, with progression to DCIS only 4 months later. Note that this detection occurred long before the formation of a discrete cancer mass, which might have been visible on an anatomic test like mammography.

\section{Differences in MCA}

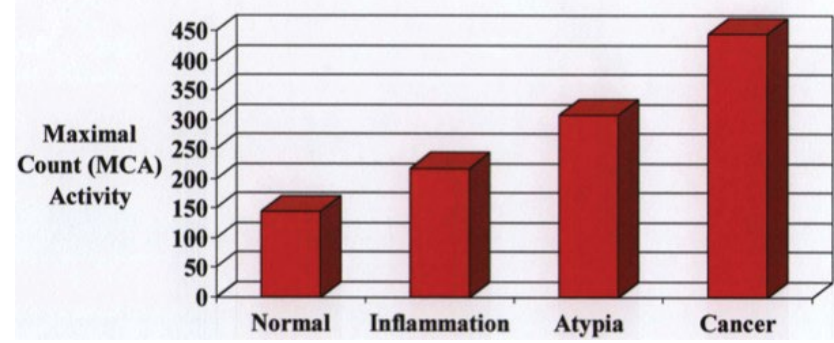

Figure 10: Results of MCA show a progression of tissue proliferation with increasing metabolic and angiogenic changes as breast tissue changes from normal to FCD to cellular atypia to breast cancer.

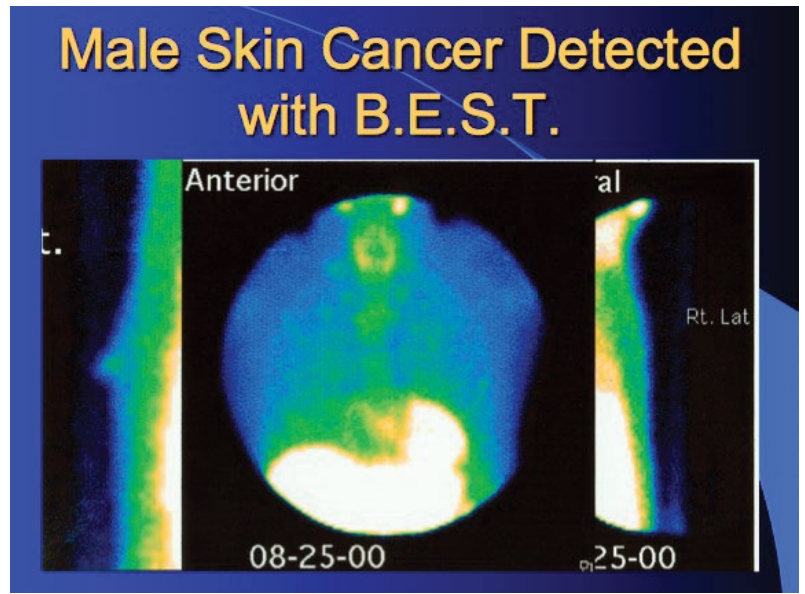

Figure 11: A male participant shows a breast cancer in the left nipple with an MCA value of 462 .

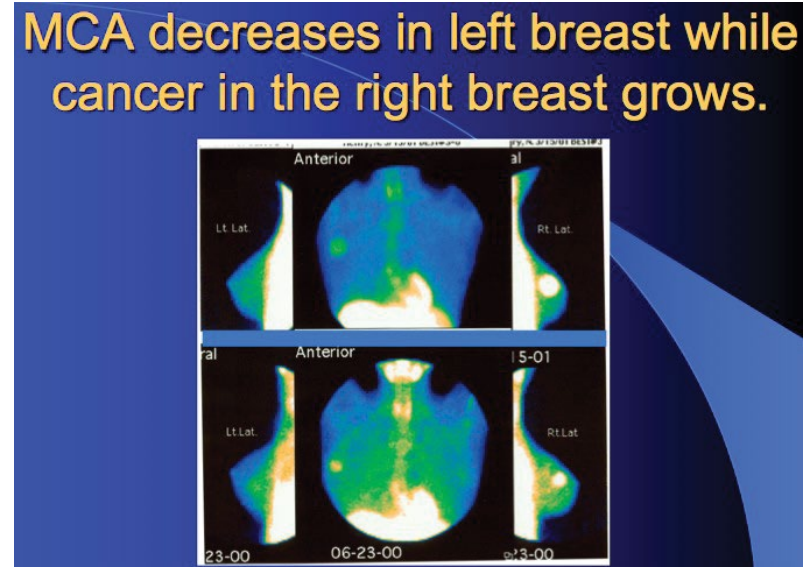

Figure 12: Over the course of 9 months, the primary cancer growing in the right breast (far right panels) showed increased MCA and an obvious increase is mass size (top panel from March of 2001) over time, with the bottom panel revealing the results from June of 2000 . The decrease in MCA of the left breast (far left panels) in this case was associated with the visual appearance of less pronounced isotope activity. The actual MCA values are discussed in the body of the original manuscript.

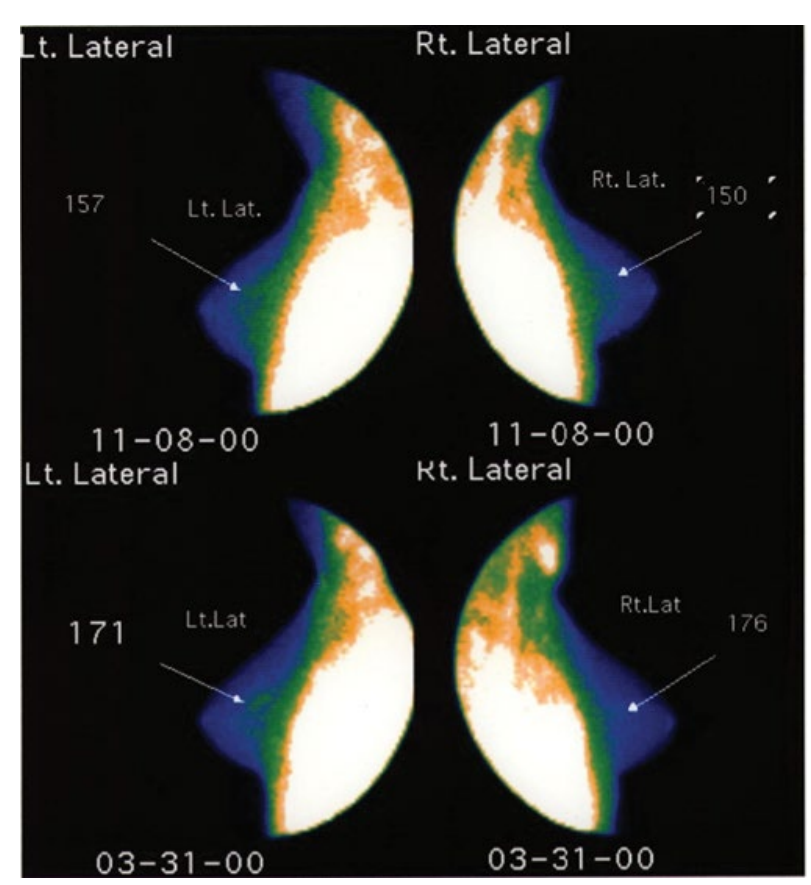

Figure 13: Improvement in the FCD for this woman is seen by the reduction in MCA following treatment.

frequently be painful and associated with palpable masses leading to mammography, ultrasounds and breast biopsy. While B.E.S.T. is able to differentiate FCD from cancer, an additional question regarding both the ability of B.E.S.T. to monitor treatment results and the question as to a possible treatment for FCD was the focus of this study. An example is shown in Figure 13 of treatment results for a woman taking a soy product for one year who showed improvement in the FCD with a reduction in the MCA while taking the product. It should be noted however that other women have shown a progression of disease 
Citation: Fleming RM, Dooley WC, Chaudhuri TK (2017) The Development of FMTVDM-BEST IMAGING@®: The Answer for Breast Cancer. Breast Enhanced Scintigraphy Test (BESTOP): Quantifying the Detection of Breast Cancer and its Treatment. J Nucl Med Radiat Ther 8: 350. doi: 10.4172/2155-9619.1000350

following the use of soy products, reinforcing the importance of tailoring individual treatment based upon actual results, remembering that each person and their cancer may react differently in response to treatment. Here, ignorance is not bliss and improved life survival and treatment response can be guided using BESTC(P.

For many, but not all of the women, the results showed an improvement in the FCD as measured by improvement in MCA. While the improvement was not statistically significant (before treatment: $249.60 \pm 98.40$; after treatment: $226.98 \pm 59.22 ; \mathrm{p}=\mathrm{NS}$ ), further analysis showed that this was statistically significant in some women, with considerable variability in individual response $(\mathrm{p}<0.01)$ to treatment.

Given the ability to quantify changes in MCA indicative of treatment response in this group of women with FCD, we looked at prior study participants who had undergone treatment of their breast cancer and demonstrated the ability of BEST(C) $($ to monitor treatment response including both recurrence and successful treatment of their cancer as shown in Figure 14.

Since one of the risk factors for breast cancer is duration of unopposed estrogen levels, we next set out to determine if women taking hormone replacement therapy (HRT) demonstrated differences in proliferative breast changes as measured by MCA, which might pose a risk for subsequent development of breast cancer. Prior to doing this we looked to see if MCA changed appreciable during the course of normal menstrual cycles. If such changes were to occur, it would indicate limitations to the timing of B.E.S.T. imaging. As noted by the example shown in Figure 15, MCA values are not appreciable influenced by menstrual cycle.

To first study the effect of HRT we looked at 327 non-pregnant, non- lactating women, including 188 who were not taking HRT and 139 who were. Figure 16 shows the graphic depiction of differences in MCA within groups as described in the legend. Of greatest significance is the greater variability seen with atypia and breast cancer in women taking HRT. The study demonstrated that this was statistically significant in women $(\mathrm{p}<0.01)$ using HRT (Table 1) and having ER- (negative) tumors [6].

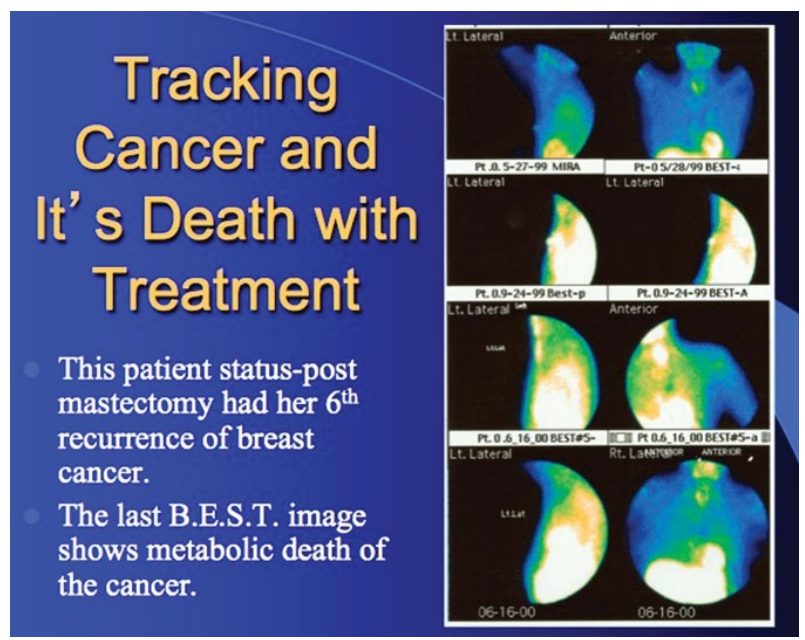

Figure 14: Over the course of time, this patient underwent both mastectomy and chemotherapy. B.E.S.TC) Imaging demonstrated that her initial treatment did not work. A change in her treatment was subsequently demonstrated to have successfully treated her breast cancer.

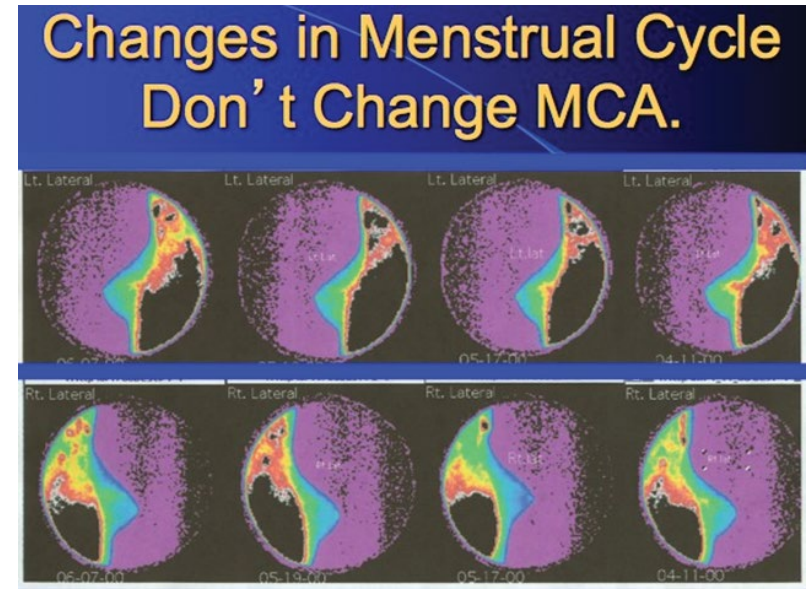

Figure 15: MCA values do not change by more than 10-15 throughout the duration of menstrual cycles.

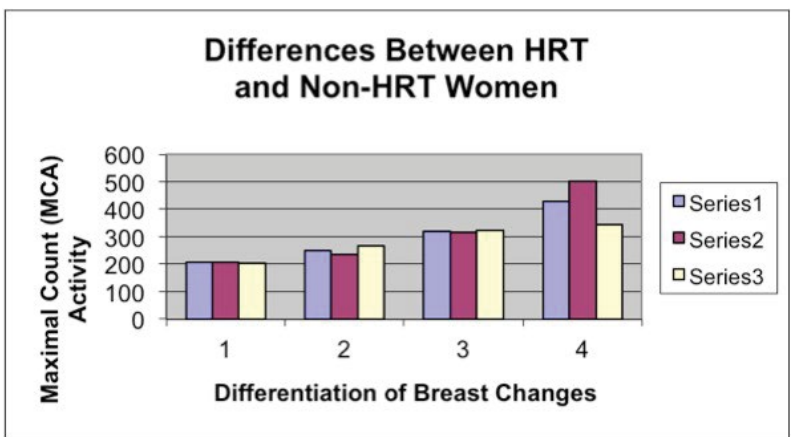

Figure 16: Group 1 represent "normal" breast tissue, group 2 are those with FCD, group 3 are those with cellular atypia, while group 4 represents those women with breast cancer. Series 1 represented all of the women, while series 2 represented those who did not take HRT and series 3 represents those who were taking HRT.

\begin{tabular}{|l|c|c|c|}
\hline Category & $\mathbf{X} \pm \boldsymbol{\sigma}$ & $\mathbf{M C A} \pm \mathbf{\sigma}$ & $\begin{array}{c}\text { Number of } \\
\text { Women }\end{array}$ \\
\hline Normal (all) & $95.27 \pm 25.85$ & $205.93 \pm 54.30$ & 141 \\
\hline Normal (-HRT) & $95.21 \pm 22.00$ & $206.74 \pm 51.15$ & 94 \\
\hline Normal (+HRT) & $95.38 \pm 32.48$ & $204.30 \pm 60.66$ & 47 \\
\hline Fiprocystc disease (all) & $114.58 \pm 29.85$ & $249.68 \pm 57.41$ & 139 \\
\hline Fibrocystic disease (-HRT) & $108.38 \pm 32.76$ & $233.88 \pm 56.16$ & 72 \\
\hline Fibrocystic disease (+HRT) & $121.26 \pm 24.93$ & $266.67 \pm 54.16$ & 67 \\
\hline Atypia (all) & $139.77 \pm 38.07$ & $320.1 \pm 100.6$ & 34 \\
\hline Atypia (-HRT) & $137.09 \pm 3454$ & $316.5 \pm 94.3$ & 15 \\
\hline Atypca (+HRT) & $141.88 \pm 41.46$ & $322.80 \pm 107.80$ & 19 \\
\hline Cancer (all) & $189.4 \pm 117.6$ & $429.0 \pm 159.0$ & 13 \\
\hline Cancer (-HRT) & $223.4 \pm 1482$ & $503.0 \pm 137.9$ & 7 \\
\hline Cancer (+HRT) & $149.7 \pm 57.5$ & $342.4 \pm 145.6$ & 6 \\
\hline X &
\end{tabular}

$\mathrm{X} \pm \sigma$ indicates average count activity (ACA) \pm the standard deviation (counts per pixel). MCA $\pm \sigma$ indicates maximal count activity (MCA) \pm the standard deviation (counts per pixel).

Table 1: Women taking HRT had a greater variability in both the ACA and MCA activity compared with women not taking HRT. This variability in tissue proliferative activity was more pronounced $(p<0.01)$ in women having cellular atypia and those having breast cancer. 
Citation: Fleming RM, Dooley WC, Chaudhuri TK (2017) The Development of FMTVDM-BEST IMAGING@®: The Answer for Breast Cancer. Breast Enhanced Scintigraphy Test (BESTOP): Quantifying the Detection of Breast Cancer and its Treatment. J Nucl Med Radiat Ther 8: 350. doi: 10.4172/2155-9619.1000350

Page 7 of 8

Table 1 shows Average Count Activity (ACA) and Maximal Count Activity (MCA) Results in BEST Imaging in 327 Women, Including 188 Not Taking Hormone Replacement Therapy (HRT) and 139 Taking HRT.

The original biopsy (tissue) studies clearly showed that women with dense breasts were just as accurately measured using BEST@( women without dense breasts. There is no increased vascularity and metabolism within the dense breast tissue to fool BEST@(P. Finally we asked if the incidence of breast cancer was higher in women taking HRT [7]. To do this we looked at 300 women, including 120 who were taking HRT and another 180 who did not take hormone therapy. The HRT study included a new addition to our BEST@(P imaging; viz. women with breast implants. Given the limitations of other imaging modalities, we were intrigued to look at the effect this might have on BEST@ $(P$ imaging. As shown in Figure 17, the breast tissue is compressed into the subcutaneous region of the chest, with the implant taking up a significant component of the imaging region. This did not affect MCA image results.

As shown in Table 2, there was a statistically significant increase in the number of women who had cellular atypia or breast cancer when they were taking HRT. This was the FIRST study to actually show this and it was proven by BEST@ $\odot$ imaging confirmed by tissue pathology. As Table 2 and Figure 18 show, MCA and tissue confirmed findings revealed there is a statistically significant increase in women having cellular atypia and breast cancer when they take HRT. In fact, the women taking HRT accounted for 10 of the 16 (62.5\%) women with

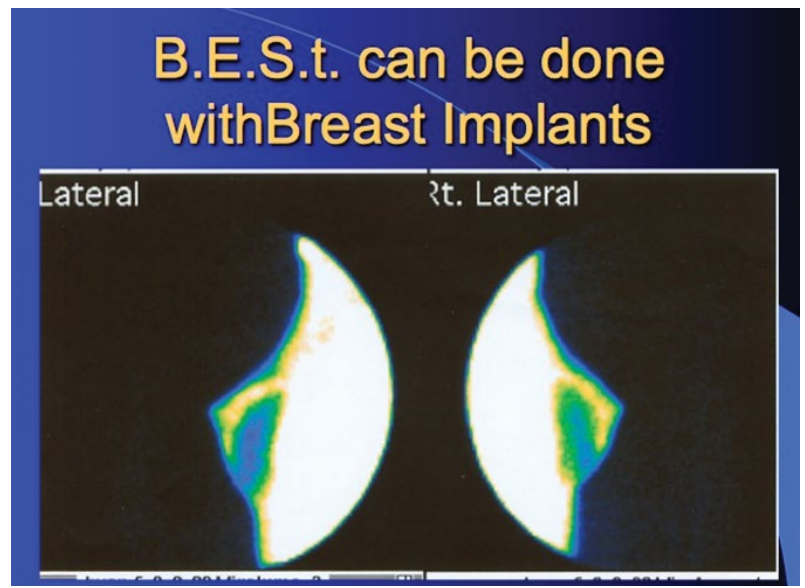

Figure 17: Breast implants compress breast tissue against the subcutaneous region of the chest. They did not result in artificial elevations in MCA values even though the qualitative appearance might appear to be more active.

\begin{tabular}{|l|c|c|c|}
\hline Category ( $\boldsymbol{n})$ & -HRT (n) & +HRT ( $\boldsymbol{n})$ & Chi-square P value \\
\hline Breast implants (8) & $75 \%(6)$ & $25 \%(2)$ & $\mathrm{P}<0.001$ \\
\hline Normal (122) & $68.8 \%(84)$ & $31.2 \%(38)$ & $\mathrm{P}<0.001$ \\
\hline Fibrocystic disease (116) & $56.9 \%(66)$ & $43.1 \%(50)$ & $\mathrm{P}=\mathrm{NS}$ \\
\hline Atypia (38) & $47.4 \%(18)$ & $52.6 \%(20)$ & $\mathrm{P}=\mathrm{NS}$ \\
\hline Cancer (16) & $37.5 \%(6)$ & $62.5 \%(10)$ & $\mathrm{P}<0.001$ \\
\hline Total women & 180 & 120 & \\
\hline$n$ is the number of women in each category. \\
\hline
\end{tabular}

Table 2: Women taking HRT demonstrated a greater incidence of both cellular atypia and breast cancer.

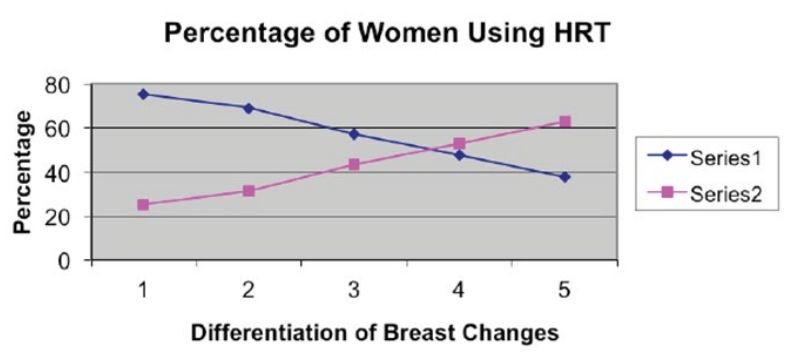

Figure 18: Series 1 represents women who did not take HRT, while series 2 represented women who took HRT. Group 1 is women with breast implants, group 2 are women with normal MCA values, group 3 had fibrocystic disease (FCD), group 4 had cellular atypia and group 5 had breast cancer.

breast cancer. Table 2 shows that women taking HRT demonstrated a greater incidence of both cellular atypia and breast cancer.

The findings of these studies, which represent eighteen (18) years of work from inception to patent were summarized and presented at a CDC sponsored conference in Greece a few years ago. The meeting was additionally represented by the then CEO (Dr. John R. Seffrin) of the American Cancer Society (ACS) who expressed an interest in this work and requested that he be kept posted on the progress of BEST@(P imaging.

The results have been presented at multiple International conferences, published as part of position papers, published in peer reviewed medical journals and medical textbook chapters 9 .

In February of 2017, the USPTO issued the patent (9566037) for these diagnostic studies marking a paradigm shift in the quantitative diagnostic imaging of heart disease and breast cancer. The method allows for detection of disease previously missed, the detection of changes leading up to cancer and critical CAD and the ability to quantitatively determine if treatment is working or if alternative treatment is indicated. This patented method is now available for licensure and distribution.

\section{Conclusion}

During its development BEST@ $(P$ has not only confirmed the fundamental differences between cancer and other types of tissue but has demonstrated the ability to accurately enhance these differences and measure them independent of the time in a woman cycle or the presence of dense breast tissue or even breast implants. BEST@(P has been able to distinguish the effects of HRT, monitor the effect of treating breast cancer and the precursors of breast cancer. It has also been able to determine whether men have breast cancer. There have been attempts by others to utilize the method to try to reproduce the results, however, without the full details provided in the license, those efforts have only shown imitation is the sincerest form of flattery.

BEST@ $\odot$ imaging presents the FIRST and only "quantitative" method, which uses tissue metabolism and vascularity resulting from angiogenesis to differentiate between calcium deposits, normal breast tissue, inflammatory changes of the breast, cellular atypia and breast cancer; both in the diagnosis and treatment responses. Further reductions in imaging times, isotope dosage and quantification of other solid tumors will soon be forthcoming. The patent includes any isotope, nuclear camera, enhancing agents and includes the use in both people and animals. 
Citation: Fleming RM, Dooley WC, Chaudhuri TK (2017) The Development of FMTVDM-BEST IMAGING@®: The Answer for Breast Cancer. Breast Enhanced Scintigraphy Test (BESTOP): Quantifying the Detection of Breast Cancer and its Treatment. J Nucl Med Radiat Ther 8: 350. doi: 10.4172/2155-9619.1000350

Page 8 of 8

\section{References}

1. Fleming RM, Harrington GM (2011) Chapter 13. Fleming harrington redistribution wash-in washout (FHRWW): The platinum standard for nuclear cardiology. Establishing better standards of care in doppler echocardiography, computed tomography and nuclear cardiology.

2. Fleming RM, Dooley WC (2002) Breast enhanced scintigraphy testing (b.e.s.t.) distinguishes between normal, inflammatory breast changes and breast cancer. a prospective analysis and comparison with mammography. Integr Cancer Ther 1: 238-245.

3. Fleming RM (2002) Mitochondrial uptake of sestamibi distinguishes between normal, inflammatory breast changes, pre-cancers and infiltrating breast cancer. Integr Cancer Thera 1: 229-237.

4. Fleming RM (2002) Breast enhanced scintigraphy test demonstrates improvement in breast inflammation in women consuming soy protein. J Nutr
132: 575 S.

5. Fleming RM (2003) What effect, if any, does soy protein have on breast tissue? Integr Cancer Ther 2: 225-228.

6. Fleming RM (2003) Are there differences in breast tissue as a result of hormone replacement therapy? Can BEST imaging distinguish these differences? Integr Cancer Ther 2: 229-234

7. Fleming RM (2003) Do women taking hormone replacement therapy (HRT) have a higher incidence of breast cancer than women who do not? Integrative Cancer Therapies 2: 235-237.

8. Fleming RM, Dooley WC (2005) Breast Enhanced Scintigraphy Test (B.E.S.T.) Imaging utilizes vascularity/angiogenesis and mitochondrial activity to distinguish between normal breast tissue, inflammation and breast cancer. $8^{\text {th }}$ International Conference. Vascular Endothelium: Translating discoveries into Public Health Practice. 